\title{
Características da agricultura familiar em município do sul do Brasil: principais enfermidades, cuidados e o uso de plantas medicinais
}

\author{
Characteristics of familiar agriculture in a municipality at South of Brazil: main illnesses, care and \\ use of medicinal plants \\ Características de la agricultura familiar en una ciudad en el sur de Brasil: principales \\ enfermedades, cuidados y uso de plantas medicinales
}

\section{Resumo}

Objetivos: Caracterizar as famílias agricultoras e identificar os problemas de saúde mais presentes, bem como, identificar se há a prática do uso de plantas medicinais no cuidado da saúde. Métodos: Estudo de caráter epidemiológico descritivo, do tipo transversal, com levantamento dos dados por inquérito domiciliar. Resultados: 104 entrevistas foram realizadas. Houve predominância de mulheres $(71,2 \%, n=74)$, com companheiro $(71,2 \%, n=74)$ e idade média de 49,2 ( $\pm 16,36)$ anos. $65,4 \%$ da população entrevistada declara ter problemas de saúde com destaque para os problemas cardíacos $(31,5 \%)$, autoimunes $(27 \%)$, doenças respiratórias $(11,7 \%)$ e de saúde mental $(10,8 \%)$. Percebe-se o uso de plantas medicinais em 79,8\%, como coadjuvantes no tratamento das enfermidades. Conclusão: $\mathrm{O}$ uso de plantas medicinais, na população rural, trata-se de uma ação comum e, geralmente, o conhecimento acerca do seu uso é transmitido através das gerações familiares. Os resultados deste estudo poderão contribuir para propor ações que envolvam desenvolvimento rural e saúde à comunidade estudada ou outras, particularmente similares, pretendendo desenvolver ações voltadas à promoção da saúde dos agricultores em situação de vulnerabilidade, ao cuidado e à prevenção de doenças.

Palavras-chave: Agricultura; Vulnerabilidade e saúde; Plantas medicinais; Área rural.

\footnotetext{
Abstract

Objectives: Characterize the agricultural families and identify the most prevalent health diseases, as well as identify the practice of using medicinal plants in health care. Methods: Descriptive epidemiological study, cross-sectional, with data collection by household survey. Results: 104 households surveys were applied. Predominance of women $(71.2 \%, n=74)$, with a partner $(71.2 \%, n=74)$ and mean age of $49.2( \pm 16.36)$ years old. $65.4 \%$ of the interviewed
} 
population declares to have health issues, highlighting heart problems $(31.5 \%)$, autoimmune (27\%), respiratory diseases $(11.7 \%)$, and mental health (10.8\%). Using medicinal plants is perceived in $79.8 \%$, as coadjuvant on illnesses treatments. Conclusion: Using medicinal plants, in rural populations, is a common action and, generally, the acknowledgment about its use is transmitted by family generations. The results of this study may contribute to propose actions that involve rural development and health to the studied community, or others, particularly similars, intending to develop actions aimed to health promotion to agricultural people in vulnerable situations, to care and prevention of diseases.

Keywords: Agriculture; Vulnerability and health; Medicinal plants; Rural area.

\section{Resumen}

Objetivos: Caracterizar las familias agricultoras e identificar los problemas de salud más presentes, así como, identificar si hay la práctica de uso de plantas medicinales en el cuidado de la salud. Métodos: Estudio epidemiológico descriptivo, transversal, con recolección de datos por encuesta de hogares. Resultados: Se realizaron 104 entrevistas. Predominó el sexo femenino $(71,2 \%, n=74)$, con pareja $(71,2 \%, n=74)$ y edad media de 49,2 $( \pm 16,36)$ años. $65,4 \%$ de la población entrevistada declaró tener problemas de salud, especialmente problemas cardíacos $(31,5 \%)$, autoinmunes (27\%), enfermedades respiratórias $(11,7 \%)$ y problemas de salud mental $(10,8 \%)$. El uso de plantas medicinales se observa en 79,8\%, como coadyuvantes en el tratamiento de las enfermedades. Conclusión: El uso de plantas medicinales, en la población rural, es una acción común y, en general, el conocimiento sobre su uso se transmite a través de las generaciones familiares. Los resultados de este estudio pueden contribuir a proponer acciones que involucren desarrollo rural y salud a la comunidad estudiada u otras, particularmente similares, con el objetivo de desarrollar acciones para promover salud de agricultores vulnerables, atención y prevención de enfermedades.

Palabras clave: Agricultura; Vulnerabilidad em salud; Plantas medicinales; Área rural.

\section{Introdução}

Segundo Neutzling (2014) o agricultor familiar possui determinado tamanho de terra que não ultrapassa cinco módulos fiscais, apresenta a renda principal obtida na agricultura e a família é a mão de obra principal utilizada na produção agrícola. Wanderley (2014) apresenta a agricultura familiar como uma forma social de produção, em que tanto os objetivos da atividade produtiva quanto o modo de organização do trabalho se apoiam na família, havendo, assim, uma estrutura produtiva que associa família-produção-trabalho, e corresponde a um modo de vida e uma cultura.

O Brasil, que apresenta junto de seus ministérios uma secretaria especial para a agricultura familiar, define em constituição o agricultor familiar aquele que pratica atividades no meio rural, atendendo, simultaneamente, aos seguintes requisitos: I - não detenha, a qualquer título, área maior do que 4 (quatro) módulos fiscais; II - utilize predominantemente mão-de-obra da própria família nas atividades econômicas do seu estabelecimento ou empreendimento; III - tenha renda familiar predominantemente originada de atividades econômicas vinculadas ao próprio estabelecimento ou empreendimento; IV - dirija seu estabelecimento ou empreendimento com sua família (Brasil, 2006a).

Segundo o Censo Agropecuário 2017 (IBGE, 2019) cerca de 3,9 milhões dos estabelecimentos agropecuários foram classificados como de agricultura familiar, o que representa $77 \%$ dos estabelecimentos levantados, sendo as principais produções o milho, a soja, o café e aqueles de origem animal, como bovinocultura de corte e leite, e avicultura. No que se refere ao estado do Rio Grande do Sul, o documento "Radiografia da Agropecuária Gaúcha 2020" apresenta os principais produtos agropecuários da região, com base no valor bruto de produção, que segue: soja, frango, arroz, bovinos, leite, milho, suínos, tabaco, fruticultura, entre outros (Rio Grande do Sul, 2020).

Ainda, cerca de 5,96 milhões de hectares foram destinados à soja, seguido de 933,17 mil hectares para o arroz, 783,30 mil hectares de milho, e apenas 126,87 mil hectares para o tabaco. Já, em relação à pecuária, os bovinos recebem maior destaque, representando 2,16 milhões de animais enviados ao abate em 2019 e 4,27 bilhões de litros de leite produzidos, enquanto a avicultura produziu 1,7 milhão de toneladas de carne de frango e 3,5 bilhões de ovos (Rio Grande do Sul, 2020).

Para Antunes (2011), investigando a integração de produtos vegetais cultivados nos estabelecimentos com até 50 hectares, com o intuito de descobrir se a produção desenvolvida no município era de cunho comercial ou de subsistência, encontrou que mais de $80 \%$ dos municípios brasileiros, produtores de trigo, e $90 \%$ dos produtores de soja, estão altamente 
integrados ao mercado, ou seja, são aqueles que vendem $90 \%$ ou mais do que produzem. Enquanto que menos de $20 \%$ dos municípios que produzem milho, arroz e mandioca são considerados como muito integrados ao mercado. Evidencia-se, assim, que muitos dos municípios gaúchos estão altamente integrados ao mercado, uma vez que, um dos principais produtos da agricultura local é a soja.

A atividade agrícola, no que tange à saúde de trabalhador, pode se constituir de forma insalubre à medida que são observados alguns riscos que os trabalhadores estão expostos, tais como: o uso de máquinas, o contato com plantas e animais em ambientes internos e externos, a exposição a variações climáticas e produtos químicos (Urio, et al., 2020), os quais podem provocar implicações agudas ou crônicas à saúde do agricultor (Bayer, 2016).

Para além da saúde ocupacional, deve-se atentar também para as comorbidades que o trabalhador rural apresenta. Em estudo realizado por Moreira, et al. (2015) constatou-se que as principais afecções entre os trabalhadores de atividade agrícola, no Brasil, foram as doenças de coluna ou costas, hipertensão arterial, artrite ou reumatismo, doenças do coração, depressão, entre outras.

O uso de plantas no tratamento e na cura de enfermidades é tão antigo quanto a espécie humana. Ainda hoje, nas regiões mais pobres do país e até mesmo nas grandes cidades brasileiras, plantas medicinais são comercializadas em feiras livres, mercados populares e encontradas em quintais residenciais (López, 2006).

O conhecimento sobre plantas medicinais simboliza, muitas vezes, o único recurso terapêutico de muitas comunidades e grupos étnicos. A população brasileira, de um modo geral, guarda um saber significativo a respeito de métodos alternativos de cura das doenças mais frequentes. As comunidades tradicionais possuem uma bagagem maior sobre o assunto, porém sofrem ameaça constante devido à influência direta da medicina ocidental moderna e pelo desinteresse dos jovens da comunidade, o que pode interromper assim o processo de transmissão do saber entre as gerações, fundamental nesse tipo de conhecimento (Amorozo, 1996).

Para Amorozo (1996), o conhecimento tradicional sobre o uso das plantas é vasto e, em muitos casos, o único recurso terapêutico disponível às populações rurais de países em desenvolvimento, tais como o Brasil. Dessa forma, o uso de plantas medicinais pode ser influenciado pela questão econômica, pelo alto custo dos medicamentos, pela dificuldade de locomoção daqueles que residem em áreas rurais ou pela tendência atual de utilização dos recursos naturais como alternativa aos medicamentos sintéticos (Battisti, et al., 2013).

Nesse contexto, a fim de aprofundar os conhecimentos acerca do agricultor familiar da região Sul do Brasil, suas condições de saúde e os meios utilizados para sanar suas enfermidades, os objetivos deste trabalho foram conhecer o perfil sociodemográfico de agricultores familiares da região, identificar os problemas de saúde mais presentes, bem como, identificar se há a prática do uso de plantas medicinais no cuidado da saúde, bem como o tipo de planta utilizada.

\section{Metodologia}

Esta pesquisa caracteriza-se por ser um estudo de caráter epidemiológico descritivo, do tipo transversal, com levantamento dos dados por inquérito domiciliar (Merchán-Hamann \& Tauil, 2021).

A pesquisa foi realizada no município de Pelotas, situado na região sul do estado do Rio Grande do Sul. A escolha do município ocorreu tendo em vista a extensa zona rural e o número significativo de agricultores em estado de vulnerabilidade nessas regiões. Assim sendo, a Colônia Maciel $-8^{\circ}$ Distrito de Pelotas - por suas características atuais se enquadra perfeitamente nos critérios inclusivos da pesquisa. A Colônia Maciel, conhecida pelo êxito da cultura do pêssego na década de 60, perdeu espaço na fruticultura para a produção de fumo no início da década de 80, atividade que se mantém presente até o momento, e sempre dividiu espaço com a produção leiteira. A produção leiteira, por sua vez, oriunda do cooperativismo, levou 
a possibilidade de produção aviária até os agricultores. Infelizmente, após desmedidos esforços e grandiosos investimentos, a cooperativa de produção de leite e aves abriu falência (Neutzling, 2014).

A população do estudo foi constituída por famílias de agricultores do local e o levantamento do número de famílias foi realizado por meio da ficha A de cadastramento das famílias encontrados na Unidade Básica de Saúde da Colônia Maciel, que apresenta uma equipe da Estratégia e Saúde da Família. A ficha A é um documento padronizado para cadastro das famílias em sua área de abrangência, a qual deve ser preenchida pelo Agente Comunitário de Saúde com dados de identificação, cadastro de todos os membros familiares, situação de moradia e saneamento (Brasil, 2003).

Para o cálculo do tamanho amostral, aplicou-se a fórmula para populações finitas, com índice de confiança de 95\%, e erro amostral de 10\%. Sendo 1034 o total de fichas cadastradas na Unidade Básica de Saúde, considerou-se esse o tamanho da população local, assim, a amostra selecionada foi de 100 participantes, e responderam ao inquérito domiciliar 104 indivíduos.

Para seleção dos entrevistados foi utilizada amostragem aleatória simples (Mahaluça, 2016), ou seja, cada participante teve chance igual e independente de ser selecionado.

Os critérios de inclusão utilizados para as famílias serão os seguintes: estar cadastrado na Estratégia e Saúde da Família; ser agricultor familiar em estado de vulnerabilidade. O estado de vulnerabilidade será considerado mediante resultado da Escala de Coelho (Escala de Risco Familiar), sendo o uso da mesma orientado pelo Ministério da Saúde junto às atividades da Estratégia de Saúde da Família. Sempre que não existir esta informação clara e precisa junto à família selecionada para a entrevista, a escala foi aplicada pela pesquisadora e a partir do resultado da mesma, decidiu-se sobre a continuidade da entrevista. Foram considerados vulneráveis todos aqueles que obtiveram escore final maior que 5 e consideravam-se responsáveis pela família. Os critérios de exclusão para os responsáveis serão os seguintes: não estar exercendo a atividade de agricultor no momento da coleta de dados; não ser encontrado o responsável pela família no domicílio após três tentativas de visita.

A coleta dos dados ocorreu entre os meses de setembro e dezembro de 2018. Os dados coletados nos questionários foram digitados em planilhas no software Microsoft Excel (2013) e, logo após, exportados para o banco de dados do programa SPSS 25.0 (Statistical Package for the Social Sciences, IBM, 2017). Para análise dos dados foi utilizado a estatística descritiva com médias e desvio padrão para as variáveis numéricas e frequências para as variáveis categóricas. Aplicou-se também o teste ANOVA para comparação de médias entre os diferentes grupos, utilizou-se como estatisticamente significativo o valor de $\mathrm{p}<0,05$.

Foram respeitados os princípios éticos da pesquisa envolvendo seres humanos, conforme a Resolução 466/12 (Brasil, 2012). O projeto de pesquisa foi encaminhado ao Comitê de Ética em Pesquisa na Área da Saúde (CEPAS/FURG) e com o parecer favorável deste deu-se início à coleta dos dados.

\section{Resultados}

Do total dos entrevistados, $71,2 \%$ (74) são mulheres e $28,8 \%$ (30) são homens. Do total, $71,2 \%$ (74) alegam viver com companheiro. A idade média da população pesquisada é de 49,2 ( $\pm 16,36)$ anos e a média de escolaridade dos mesmos é de $7,1( \pm 4,59)$ anos. A composição familiar dos pesquisados apresenta, em média, $3,1( \pm 1,18)$ membros. A renda familiar média mensal dos agricultores entrevistados é de $2,2( \pm 1,88)$ salários-mínimos e 45,2\% (47) tem renda exclusiva das atividades agrícola e pecuária. Dos que vivem exclusivamente da agropecuária, 31,9\% (15) se declaram satisfeitos com suas atividades, $12,8 \%$ (6) dos entrevistados declararam-se não satisfeitos com a agricultura, $48,9 \%$ (23) pouco satisfeitos e 6,4\% (3) não responderam. Vale ressaltar que, do todo, 26,9\% (28) sentiam-se satisfeitos com a agricultura, 9,6\% (10) não satisfeitos, 40,4\% (42) declararam estarem pouco satisfeitos, e 23,1\% (24) não responderam à pergunta. A área média total das propriedades visitadas é de $15,7( \pm 27,49)$ hectares (Quadro 1). 
Quadro 1: Características socioeconômicas da população pesquisada.

\begin{tabular}{|c|c|c|}
\hline & $\mathbf{n}$ & $\%$ \\
\hline \multicolumn{3}{|l|}{ Gênero } \\
\hline Feminino & 74 & 71,2 \\
\hline \multicolumn{3}{|l|}{ Origem da renda } \\
\hline Agricultura e pecuária & 47 & 45,2 \\
\hline \multicolumn{3}{|l|}{ Satisfação com agricultura } \\
\hline Satisfeitos & 28 & 26,9 \\
\hline Pouco satisfeitos & 42 & 40,4 \\
\hline Não satisfeitos & 10 & 9,6 \\
\hline Não respondeu & 24 & 23,1 \\
\hline Agropecuária & 60 & 57,7 \\
\hline Não responderam & 15 & 14,4 \\
\hline \multicolumn{3}{|l|}{ Uso de agrotóxicos } \\
\hline Sim & 51 & 49,0 \\
\hline Não & 48 & 46,2 \\
\hline \multirow[t]{2}{*}{ Não respondeu } & 5 & 4,8 \\
\hline & Média & DP \\
\hline Idade em anos & 49,2 & 16,36 \\
\hline Escolaridade em anos & 7,1 & 4,59 \\
\hline
\end{tabular}

DP: desvio padrão. Fonte: Dados da pesquisa.

A agricultura é atividade presente em 73,1\% (76) das famílias de agricultores entrevistadas. O principal cultivo é o pêssego, presente em 53,9\% (41) dentre os cultivos dos entrevistados, essa cultura ocupa uma área média de 5,2 ( $\pm 4,99)$ hectares nas propriedades participantes da pesquisa. As hortas orgânicas são cultivadas em 17,1\% (13) das propriedades entrevistadas e ocupam uma área média de 0,3 $( \pm 0,39)$ hectares em cada propriedade. O cultivo de milho aparece em 35,5\% (27) das propriedades visitadas e utiliza uma área média de $1,5( \pm 1,35)$ hectares. O cultivo de feijão e frutas e vegetais variados estão presentes em $22,4 \%$ (17) e 37,9\% (29) das propriedades visitadas, e ocupam áreas médias de 0,5 ( $\pm 0,38)$ e 0,7 ( $\pm 0,91)$ hectares, respectivamente. As batatas representam 11,8\% (9) dos cultivos nas propriedades visitadas e utilizam uma área média de $0,2( \pm 0,26)$ hectares. A soja é cultivada em 9,2\% (7) das propriedades que participaram da pesquisa e utilizam área média de $15,8( \pm 10,46)$ hectares. E o fumo é cultivo presente em 5,3\% (4) das propriedades visitadas, ocupando área média de 2,7 $( \pm 2,06)$ hectares. Quando comparadas as áreas de cultivos, observou-se que a soja apresenta maior extensão de área cultivada $(\mathrm{p}<0,05)$, a extensão de área cultivada pelo pêssego é menor quando comparada à extensão de área cultivada de soja $(\mathrm{p}<0,005)$ e maior quando comparada aos demais cultivos $(\mathrm{p}<0,005)$. Dentre os demais cultivos não houveram diferenças estatisticamente significativas quando comparadas as extensões de áreas utilizadas (Figura 1). 
Figura 1: Extensão média dos cultivos em hectares.

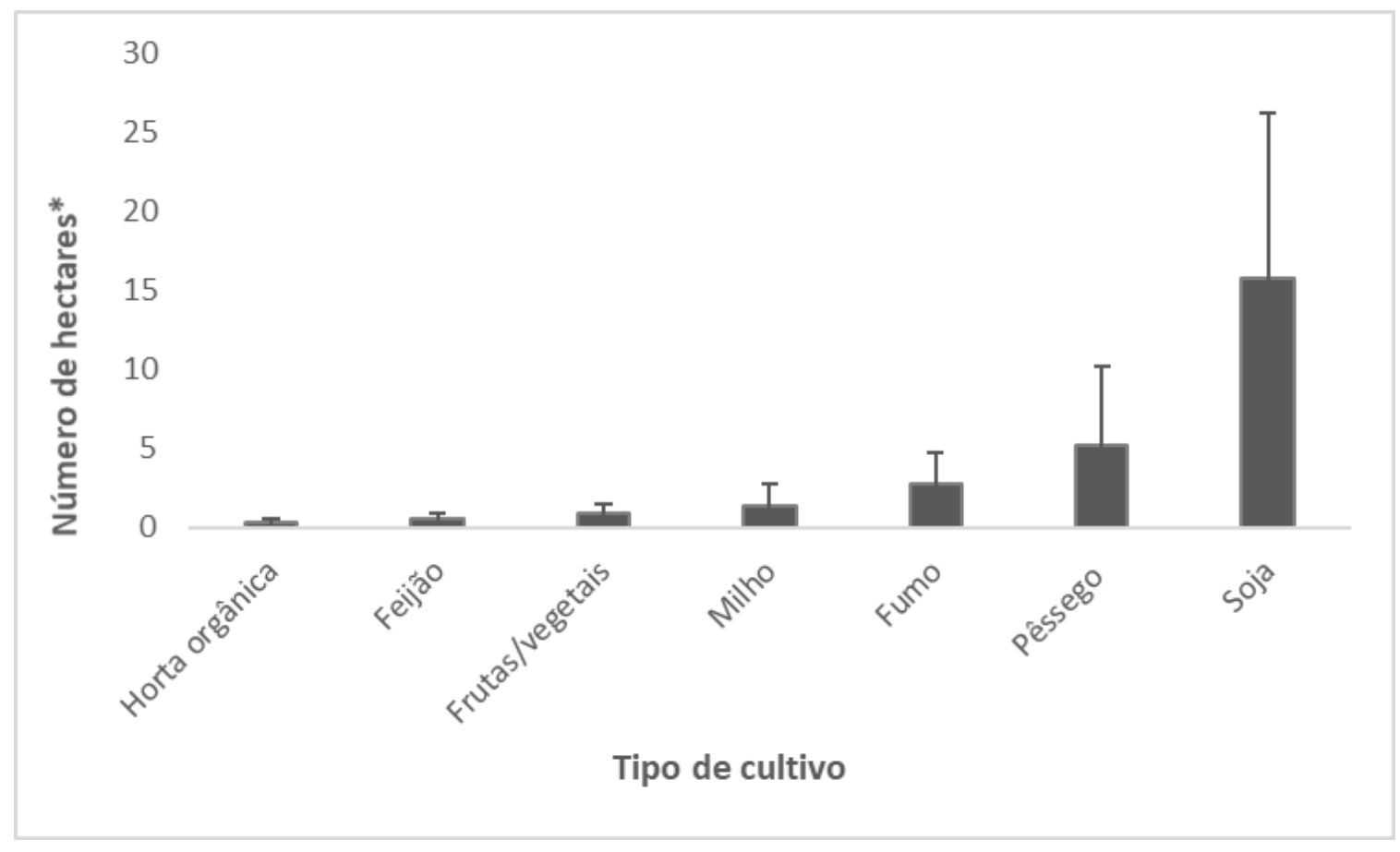

*Teste ANOVA p<0,05. Fonte: Dados da pesquisa.

Do total de agricultores familiares entrevistados 70,2\% (73) criam animais. As aves são criadas em $80,8 \%$ (59) das propriedades visitadas e a média do rebanho é de $29,5( \pm 30,22)$ animais. Os bovinos estão presentes em $67,1 \%$ (49) das propriedades participantes da pesquisa e seu rebanho médio é de $8( \pm 11,8)$ animais. Os suínos são criados em 35,6\% (26) das propriedades pesquisadas e apresentam rebanho médio de $3( \pm 2,03)$ porcos. Cabras e ovelhas são criadas em 13,6\% (10) das propriedades, e seus rebanhos são, respectivamente, de $5,8( \pm 2,58)$ e $15,4( \pm 13,18)$ animais. Outros animais são criados em 10,9\% (8) das propriedades visitadas.

Em relação ao uso de agrotóxicos, 49,0\% dos entrevistados utilizam agrotóxicos em seus cultivos (Quadro 1). Dentre esta população, encontram-se, em totalidade, os produtores de pêssego, soja e fumo.

O total de 65,4\% (68) da população entrevistada declara ter problemas de saúde. Dentre os problemas de saúde os principais relatados foram: problemas cardíacos $(31,5 \%)$, autoimunes $(27 \%)$, doenças respiratórias $(11,7 \%)$ e de saúde mental (10,8\%), como pode ser observado na Figura 2. 
Figura 2: Principais problemas de saúde da população pesquisada.

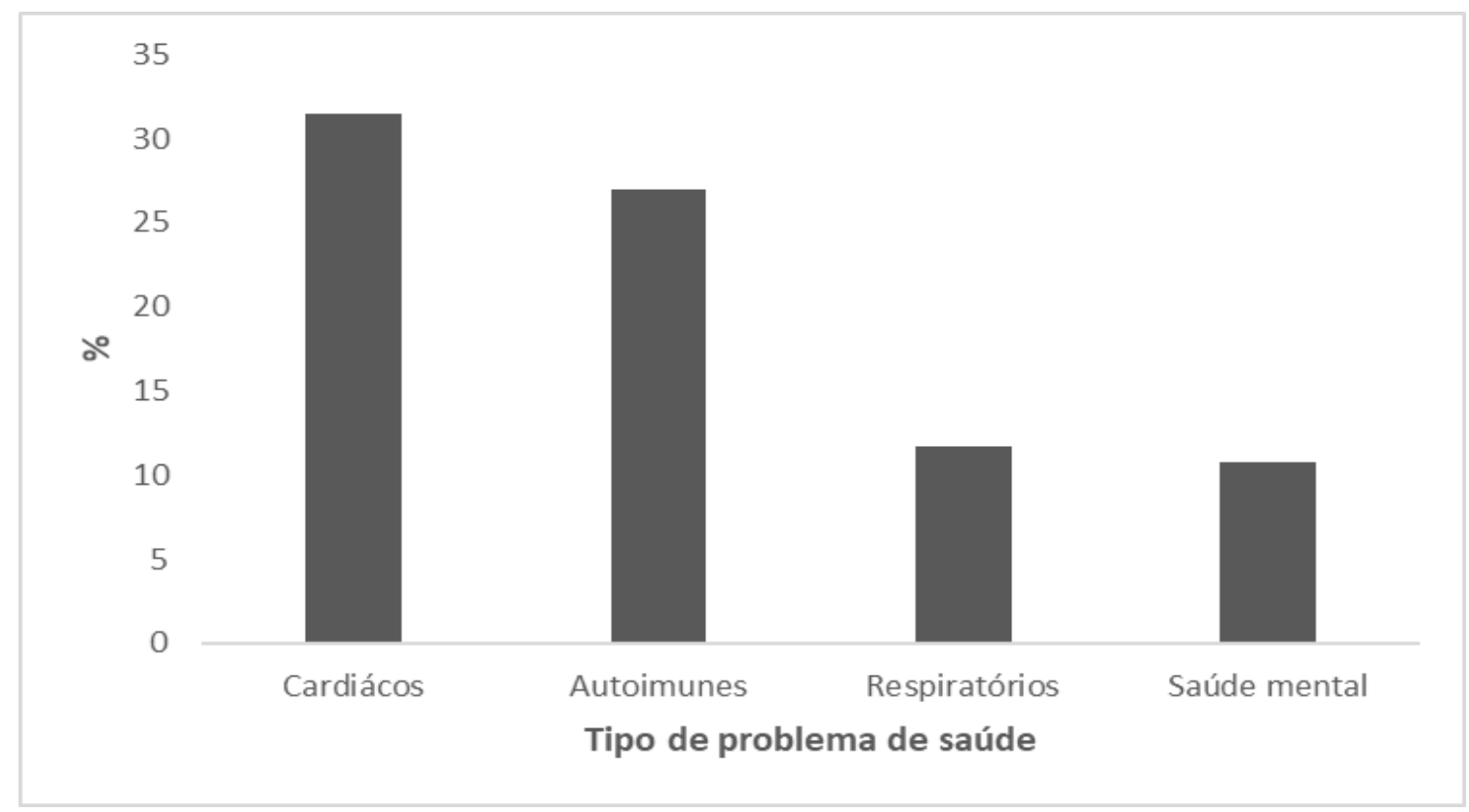

Fonte: Dados da pesquisa.

Isto posto, a população pesquisada foi perguntada sobre suas diferentes formas de cuidado e $71,2 \%$ declaram ir à Unidade Básica de Saúde local quando necessita de algum cuidado de saúde, o restante alega o autocuidado. No que se refere ao uso de medicações, 70,2\% dos entrevistados fazem uso somente de medicação prescrita pelo profissional de saúde e 53,8\% dos entrevistados fazem uso de medicação prescrita ou não pelo profissional de saúde para tratar suas doenças.

Ainda sobre formas de cuidado, $79,8 \%$ da população participante da pesquisa declara fazer uso de plantas medicinais como coadjuvantes no tratamento de enfermidades. Dentre as plantas medicinais mais utilizadas destacam-se: marcela (Achyrocline satureioides), utilizada por 15,9\% dos entrevistados; funcho (Foeniculum vulgare), utilizado por $7 \%$ dos participantes da pesquisa; camomila (Matricaria chamomilla) utilizada por 6,5\% dos participantes entrevistados; tansagem (Plantago major) e cidreira (Melissa officinalis) são utilizados por 5,6\% dos entrevistados cada; boldo (Peumus boldus), é utilizado por 5,1\% dos participantes; e malva (Malva sylvestris), é utilizada por 4,2\% dos pesquisados (Quadro 2).

Quadro 2: Principais plantas medicinais utilizadas pela população pesquisada.

\begin{tabular}{|l|l|l|}
\hline Planta medicinal & $\mathbf{n}$ & $\%$ \\
\hline Marcela & 34 & 15,9 \\
\hline Funcho & 15 & 7,0 \\
\hline Camomila & 14 & 6,5 \\
\hline Tansagem & 12 & 5,6 \\
\hline Cidreira & 12 & 5,6 \\
\hline Boldo & 11 & 5,1 \\
\hline Malva & 9 & 4,2 \\
\hline
\end{tabular}

Fonte: Dados da pesquisa. 


\section{Discussão}

Considerando as características sociodemográficas, nota-se predominância de mulheres $(71,2 \%, \mathrm{n}=74)$, que vivem com companheiro $(71,2 \%, n=74)$, com idade média de 49,2 $( \pm 16,36)$ anos e renda familiar mensal de $2,2( \pm 1,88)$ saláriosmínimos. Assemelha-se ao estudo de Battisti, et al. (2013), onde encontraram 90\% de mulheres, com mais de 55 anos de idade, e renda salarial inferior a três salários-mínimos, e de Ceolin, et al. (2011), que também encontraram maioria feminina, porém mais jovens (entre 20 e 39 anos), e $63 \%$ dos integrantes com ensino fundamental incompleto, que coincide ao presente estudo [média de escolaridade de 7,1 ( $(4,59)$ anos], uma vez que, tem-se por lei duração de nove anos para o ensino fundamental (Brasil, 2006b). Da mesma forma, Gonçalves e Pasa (2015), em estudo realizado na Comunidade Sucuri, distrito de Cuiabá MT, encontraram semelhanças com esta pesquisa, como maioria feminina, em matrimônio, com variação de idade entre 25 e 84 anos, renda mensal de um a três salários-mínimos, as quais também expuseram diferenças, no quesito escolaridade, já que sua maioria possuía ensino fundamental completo.

No tocante à agropecuária, a agricultura esteve presente em 73,1\% (76) das famílias de agricultores entrevistadas, enquanto a pecuária em 70,2\% (73). As principais culturas agrícolas foram de pêssego, frutas e vegetais variados, de milho, feijão, de hortas orgânicas, batatas, soja e fumo. Já na pecuária foram as aves, os bovinos, os suínos, cabras e ovelhas, e demais espécies de animais.

Tais dados, diferem do estudo de Garcez, et al. (2020), realizado no Rio Grande do Sul com 20 agricultores familiares, aos quais encontraram como atividades agrícolas de maior frequência a produção de grãos, seguida da produção de subsistência, bovinocultura de leite, criação de frango, produção de fumo e arrendamento. Em outro estudo gaúcho, a produção agrícola apresentada, ainda diverge dos demais estudos, uma vez que, a região caracterizava-se pela fruticultura, com destaque para uva e maçã; além de produção de milho, feijão e, frutas e vegetais variados. A pecuária era centrada em aves, bovinos e suínos (Faria, et al., 2000). Nesse contexto, percebe-se que mesmo tratando-se de um único estado, a diversidade produtiva na agricultura é bastante evidente.

Neste atual trabalho, 49,0\% dos entrevistados referem utilizar agrotóxicos em seus cultivos, sendo, que os produtores de pêssego, soja e fumo, encontram-se em totalidade. Em um estudo, realizado na zona rural de Vitória da Conquista - BA, com 31 agricultores familiares, foi identificado que cerca de $29,0 \%$ dos entrevistados faziam uso de agrotóxicos na produção agrícola, sendo os principais cultivos o feijão, milho e mandioca (Oliveira, et al., 2021).

Soglio e Kubo (2016), por sua vez, afirmam que o uso de agrotóxicos, de sementes geneticamente modificadas e de insumos químicos ocorre em maior escala na manutenção de monoculturas como a de trigo, arroz, milho, algodão e soja.

Corroborando às demais pesquisas, no ano de 2015, a soja foi a cultura que mais utilizou agrotóxicos (63\%), seguido do milho $(13 \%)$ e da cana-de-açúcar (5\%). Juntos, estes três cultivos representaram $76 \%$ de toda a área plantada do Brasil (Pignati, et al., 2017). Nesse sentido, possivelmente, seja essa a razão de quase metade dos agricultores fazerem uso de agrotóxicos, uma vez que, dentre os principais cultivos neste estudo, estão o milho, soja e feijão.

Kotz, et al. (2021) apresentam que práticas laborais de agricultores vêm sendo desenvolvidas de maneira insegura devido ao uso inadequado de equipamentos de proteção individual. Ainda, a exposição humana aos agrotóxicos representa grave problema da saúde pública, pois o impacto da exposição nem sempre é reconhecido como a causa das enfermidades e por isso, essas demandas tendem a serem inviabilizadas e não respondidas no âmbito dos serviços de saúde.

Em estudo realizado com 29 trabalhadores, de seis propriedades agrícolas do município de Lagoa Seca - PB, que buscou verificar indicadores de qualidade de vida do agricultor e em que medida as atividades podem influenciar nos agravos à saúde, constatou-se que o domínio físico foi identificado como contribuinte positivo para a qualidade de vida com a faceta de capacidade para o trabalho, no entanto, 62\% dos agricultores relataram sentir alguma dor/desconforto durante a jornada de trabalho, especialmente, problemas relacionados ao aparelho osteomuscular (48\%), ao aparelho cardiovascular (24\%), do 
aparelho respiratório (9\%) e do aparelho endócrino (19\%) (Pessoa \& Alchieri, 2018). Tais porcentagens, aproximam-se às encontradas no presente estudo, principalmente, aos problemas cardíacos $(31,5 \%)$ e respiratórios $(11,7 \%)$.

À vista disso, o uso de plantas medicinais, principalmente, em comunidades rurais, passa a ser aliado no tratamento de enfermidades, já que, configura-se como um patrimônio tradicional da comunidade camponesa (Rückert, et al., 2018). Nesta pesquisa, 79,8\% dos participantes declararam fazer uso de plantas medicinais como coadjuvantes do tratamento de patologias, sendo as principais a marcela (Achyrocline satureioides), o funcho (Foeniculum vulgare), a camomila (Matricaria chamomilla), a tansagem (Plantago major), a cidreira (Melissa officinalis), o boldo (Peumus boldus) e a malva (Malva sylvestris). Battisti, et al. (2013), que também estudaram o uso de plantas medicinais no estado gaúcho, encontraram prevalência de diferentes espécies medicinais como o poejo (Mentha pulegium), o guaco (Mikania glomerata), a cavalinha (Equisetum sp.) e a manjerona (Origanum majorana), e outras em comum, como a marcela, a camomila, a tansagem e o boldo. No entanto, quando o foco está em outro estado brasileiro, como São Paulo, diferentes plantas medicinais são nomeadas, como a erva Santa Maria (Dysphania ambrosioides), a erva cidreira, o boldo, a hortelã, a caferana (Tachia guyanensis), a arruda e o poejo (Bueno \& Sant'Ana, 2014).

Salienta-se que espécies medicinais, como a marcela tem maior ocorrência em estudos gaúchos, uma vez que, é tida como planta símbolo do estado (Rio Grande do Sul, 2002). Enquanto o boldo, nativo da África Tropical, foi introduzido no Brasil no período colonial e facilmente cultivado em hortas e jardins domésticos do país (Fernandes, et al., 2021), por isso sua presença em todos os artigos mencionados.

O reconhecimento e o resgate do saber local sobre as plantas medicinais são fundamentais em comunidades rurais, pois os remédios caseiros surgem como alternativa de cura, muitas vezes, a única devido à falta de outros recursos para cuidar da saúde (Cunha \& Bortolotto, 2011).

\section{Considerações Finais}

O presente artigo buscou caracterizar as famílias agricultoras e identificar os problemas de saúde mais presentes, bem como, identificar se há a prática do uso de plantas medicinais no cuidado da saúde e suas variedades.

Nota-se que, durante o estudo, mais da metade da população $(65,4 \%)$ apresentava algum problema de saúde e um número, ainda mais expressivo, fazia uso de plantas medicinais como coadjuvante no tratamento das enfermidades $(79,8 \%)$. Ademais, é importante ressaltar que o uso de plantas medicinais, na população rural, trata-se de uma ação comum e, geralmente, o conhecimento acerca do uso é transmitido através das gerações familiares.

Ainda que o intuito fosse aprofundar os conhecimentos sobre o agricultor familiar na região Sul, suas condições de saúde e os meios utilizados para sanar suas enfermidades, percebe-se um déficit na produção científica atual, que permita uma discussão mais abrangente sobre o tema.

Os resultados deste estudo poderão contribuir para propor ações que envolvam desenvolvimento rural e saúde à comunidade estudada ou outras, particularmente similares, pretendendo desenvolver ações voltadas à promoção da saúde dos agricultores em situação de vulnerabilidade, ao cuidado e à prevenção de doenças.

\section{Referências}

Amorozo, M. C. M. (1996). A abordagem etnobotânica na pesquisa de plantas medicinais. In Distasi, L. C. (Org.). Plantas medicinais: arte e ciência, um guia de estudo interdisciplinar (pp. 47-68). EDUSP.

Antunes, D. S. (2011). Características da agricultura familiar. In Instituto Brasileiro de Geografia e Estatística, Atlas do espaço rural brasileiro (pp. 113-133). IBGE. https://biblioteca.ibge.gov.br/visualizacao/livros/liv63372_cap5.pdf.

Battisti, C., Garlet, T. M. B., Essi, L., Horbach, R. K., Andrade, A., \& Badke, M. R. (2013). Plantas medicinais utilizadas no município de Palmeira das Missões, RS, Brasil. R. bras. Bioci., 11(3):338-348. http://www.ufrgs.br/seerbio/ojs/index.php/rbb/article/view/2457. 
Bayer, L. J. Z. (2016). Os agravos à saúde do trabalhador rural. (Trabalho de Conclusão de Curso). Universidade do Estado do Rio de Janeiro, Rio de Janeiro, RJ. https://ares.unasus.gov.br/acervo/html/ARES/8077/1/LORHAYNNE\%20DE\%20JESUS\%20ZEBENDE\%20BAYER.pdf.

Brasil. (2006a). Lei $n^{o} 11.326$, de 24 de julho de 2006. http://www.planalto.gov.br/ccivil_03/_ato2004-2006/2006/lei/111326.htm.

Brasil. (2006b). Lei $n^{o} 11.274$, de 6 de fevereiro de 2006. https://www.planalto.gov.br/ccivil_03/_ato2004-2006/2006/lei/111274.htm.

Brasil. Ministério da Saúde. (2012). Resolução $n^{o} \quad 466$, de 12 de dezembro 2012 . https://bvsms.saude.gov.br/bvs/saudelegis/cns/2013/res0466_12_12_2012.html.

Brasil. Ministério da Saúde. Secretaria de Atenção à Saúde. Departamento de Atenção Básica. (2003). SIAB: manual do sistema de Informação de Atenção Básica. Brasília: Ministério da Saúde. http://www2.datasus.gov.br/SIAB/siab/downloads/manual.pdf.

Bueno, A. R., \& Sant'Ana, A. L. (2014). Conhecimento, cultivo e formas de utilização de plantas medicinais por agricultores familiares de Santa Albertina (SP). Cultivando o Saber, 7(4):319-331. https://www.fag.edu.br/upload/revista/cultivando_o_saber/54eb29a0da96a.pdf.

Ceolin, T., Heck, R. M., Barbieri, R. L., Schwartz, E., Muniz, R. M., \& Pillon, C. N. (2011). Plantas medicinais: transmissão do conhecimento nas famílias de agricultores de base ecológica no Sul do RS. Rev Esc Enferm https://www.scielo.br/j/reeusp/a/RhYtqkRwFSRDYBR6gGqZhxM/?format=pdf \& lang=pt.

Cunha, S. A., \& Bortolotto, I. M. (2011). Etnobotânica de Plantas Medicinais no Assentamento Monjolinho, município de Anastácio, Mato Grosso do Sul, Brasil. Acta Botanica Brasilica, 25(3):685-698. https://www.scielo.br/j/abb/a/tJ34ptrvCff8NR9ZBJP99Jj/?format=pdf\&lang=pt.

Faria, N. M. X., Facchini, L. A., Fassa, A. G., \& Tomasi, E. (2000). Processo de produção rural e saúde na serra gaúcha: um estudo descritivo. Cad. Saúde Pública, 16(1):115-128. https://www.scielo.br/j/csp/a/HW5KHvQkLQj5dwhYG59wWXF/?lang=pt\&format=pdf.

Fernandes, J. M., Lopes, C. R. A. S., \& Almeida, A. A. S. D. (2021). Morfologia de espécies medicinais de boldo cultivadas no Brasil. Research, Society and Development, 10(6):e42910615824. http://dx.doi.org/10.33448/rsd-v10i6.15824.

Garcez, J. C., Silva, D. M., Hengles, A. C. V., Guerra, D., Bisognin, R. P., \& Bohrer, E. G. (2020). Análise da qualidade de vida de agricultores familiares: estudo de caso em Três Passos, Rio Grande do Sul (RS). Nativa, Sinop, 8(4):506-513. https://doi.org/10.31413/nativa.v8i4.9826.

Gonçalves, K. G., \& Pasa, M. C. (2015). A etnobotânica e as plantas medicinais na Comunidade Sucuri, Cuiabá, MT, Brasil. Interações, 16(2):245-256. doi: http://dx.doi.org/10.1590/1518-70122015201.

IBM SPSS Statistics for Windows, versão 25.0. (2017). IBM Corporation.

Instituto Brasileiro de Geografia e Estatística. (2019). Censo Agropecuário 2017: Resultados definitivos. 8, 1-105. IBGE. https://biblioteca.ibge.gov.br/visualizacao/periodicos/3096/agro_2017_resultados_definitivos.pdf.

Kotz, E. J., Cabral, F. B., Trezzi, I., Dias, G. L., Spanevello, R. M., Hildebrandt, L. M. \& Silveira, A. (2021). Noções e uso de agrotóxicos: Um estudo de caso com agricultores familiares. Research, Society and Development, 10(7): e50510716898. http://dx.doi.org/10.33448/rsd-v10i7.16898.

López, C. A. A. (2006). Considerações gerais sobre plantas medicinais. Revista Ambiente: Gestão e Desenvolvimento, 1(1):19:27. https://pt.scribd.com/document/151508250/consideracoes-gerais-sobre-plantas-medicinais.

Mahaluça, F. A. (2016). Noções de Amostragem. https://www.academia.edu/37979381/ESTAT\%C3\%8DSTICA_APLICADA_FILIPE_A NT\%C3\%93NIO_MAHALU\%C3\%87A.

Merchán-Hamann, E., \& Tauil, P. L. (2021). Proposta de classificação de diferentes tipos de estudos epidemiológicos descritivos. Epidemiol. Serv. Saude, 30(1):e2018126. http://doi.org/10.1590/S1679-49742021000100026.

Microsoft Excel (2013). Albuquerque, NM: Microsoft Corporation.

Moreira, J. P. L., Oliveira, B. L. C. A., Muzi, C. D., Cunha, C. L. F., Brito, A. S., \& Luiz, R. R. (2015). A saúde dos trabalhadores da atividade rural no Brasil. Cad. Saúde Pública, 31(8):1698-1708. http://dx.doi.org/10.1590/0102-311X00105114.

Neutzling, A. S. (2014). Performance of small scale milk producers in the South of Rio Grande do Sul, Brazil: the impact of herd management and milk marketing strategies. Goettingen: CUVILLIER VERLAG.

Oliveira, R. A., Souza, C. C., Anjos, D. A., Kochergin, C. N., \& Zanuto, M. E. (2021). Nível de conhecimento de agricultores familiares em relação ao uso de agrotóxicos em um município do interior da Bahia. Rev. Saúde.Com., 17(3):2254-2266. 10.22481/rsc.v17i3.8094.

Pessoa, Y. S. R. Q., \& Alchieri, J. C. (2018). O trabalho do agricultor e as repercussões em sua saúde. Revista Espaço Acadêmico, 18(211):11-20. https://periodicos.uem.br/ojs/index.php/EspacoAcademico/article/view/44959.

Pignati, W. A., Lima, F. A. N. S., Lara, S. S., Correa, M. L. M., Barbosa, J. R. \& Pignatti, M. G. (2017). Distribuição espacial do uso de agrotóxico no Brasil: uma ferramenta para a Vigilância em Saúde. Ciência \& Saúde Coletiva, 22(10):3281-3293. 10.1590/1413-812320172210.17742017.

Rio Grande do Sul. (2002). Lei $n^{o}$ 11.858, de 5 de dezembro de 2002. http://www.al.rs.gov.br/legis/M010/M0100099.asp?H id_Tipo=TEXTO\&Hid_TodasNormas=706\&hTexto=\&Hid_IDNorma=706.

Rio Grande do Sul. Departamento de Políticas Agrícolas e Desenvolvimento Rural. (2020). Radiografia da Agropecuária Gaúcha 2020. Rio Grande do Sul. https://www.agricultura.rs.gov.br/upload/arquivos/202009/26185534-radiografia-da-agropecuaria-gaucha-2020-1.pdf. 
Research, Society and Development, v. 10, n. 16, e523101623715, 2021

(CC BY 4.0) | ISSN 2525-3409 | DOI: http://dx.doi.org/10.33448/rsd-v10i16.23715

Rückert, B., Cunha, D. M., \& Modena, C. M. (2018). Saberes e práticas de cuidado em saúde da população do campo: revisão integrativa da literatura. Interface (Botucatu), 22(66):903-914. 10.1590/1807-57622017.0449.

Soglio, F. D., \& Kubo, R. R. (2016). Desenvolvimento, agricultura e sustentabilidade (pp. 206). Porto Alegre: Editora da UFRGS. http://www.ufrgs.br/cursopgdr/downloadsSerie/derad105.pdf.

Urio, A., Souza, J. B., Geremia, D. S., Heidemann, I. T. S. B., \& Colliselli, L. (2020). Promoção da saúde do jovem agricultor familiar: perspectiva para um envelhecimento saudável. Rev. Enferm. UFSM, 10(43), 1-19. 10.5902/2179769240131.

Wanderley, M. N. B. O. (2014). Campesinato Brasileiro: uma história de resistência. RESR, 52(1), S025-S044. https://www.scielo.br/j/resr/a/4Hn3FCvFdb9VBYwSwJfKSGJ/?lang=pt\&format=pdf. 